\title{
GAUGE THEORY SCATTERING FROM THE ADS/CFT CORRESPONDENCE
}

\author{
Romuald A. Janik \\ Service de Physique Theorique CEA-Saclay \\ F-91191 Gif-sur-Yvette Cedex, France \\ and M.Smoluchowski Institute of Physics, Jagellonian University \\ Reymonta 4, 30-059 Cracow, Poland \\ janik@spht.saclay.cea.fr
}

\begin{abstract}
We use the AdS/CFT correspondence to study near forward scattering of colourless objects in gauge theory in the high energy limit. We find an unexpected from the gauge theory perspective 'gravity-like' $s{ }^{1}$ behaviour of the amplitudes coming from bulk graviton exchange.
\end{abstract}

\section{INTRODUCTION}

In this talk we would like to present results on certain aspects of high energy scattering in gauge theory from the point of view of the AdS/CFT correspondence [1,2]. The full account of these results appeared in [3]. The motivation for this study is twofold. Firstly, the duality between supergravity (string theory) on AdS and supersymmetric gauge theory allows for a completely new insight into the dynamics of gauge theory in the strongly coupled regime. Its consequences regarding the high energy limit of gauge theory have not been explored so far (see $[3,4])$. Secondly, expectations on the behaviour of high energy field theory amplitudes can shed some light on the behaviour of the string theory side and point out some phenomenae unexpected from the gravity point of view.

The outline of this talk is as follows. First we identify a suitable observable on the gauge theory side, then we apply the AdS/CFT correspondence to calculate this quantity and finally we discuss the implications of our results.

*Talk presented at the NATO ASI 'Progress in String Theory and M-theory', Cargese 1999. 


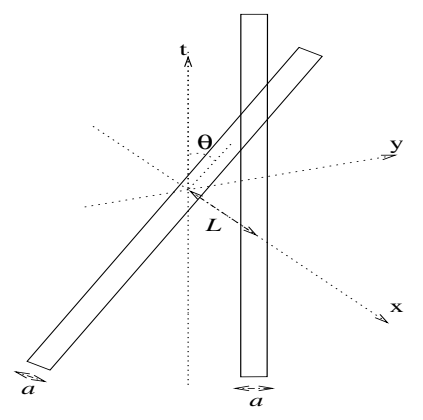

Figure 1.1 Geometry of the Wilson loops in euclidean space.

\section{GAUGE THEORY OBSERVABLE}

In this section we will define the gauge theory observable related to the near foward scattering amplitude:

$$
\frac{1}{s} A(s, t)=\int d^{2} b e^{i q b} \frac{e^{i 2 \delta(b, s)}-1}{2 i} \quad t=-q^{2}
$$

where $b$ is the impact parameter (in the following we will denote its modulus by $L$ ) and $\delta$ is the phase shift.

In QCD the scattering amplitude between quarks in the eikonal approximation (valid in the high energy $s$, fixed (and small) momentum transfer $t$ ) is given by a correlation function of two Wilson lines which follow the classical straight line quark trajectories $[5,6,7]: W_{1} \longrightarrow$ $x_{1}^{\mu}=p_{1}^{\mu} \tau$ and $W_{2} \longrightarrow x_{2}^{\mu}=x_{\perp}^{\mu}+p_{2}^{\mu} \tau$. The amplitude is in general infrared divergent and to overcome this it is convenient to consider the scattering of colourless $q \bar{q}$ pairs of transverse separation $a$. In this case the two Wilson lines are replaced by two rectangular Wilson loops each formed by the trajectories of the quark and antiquark forming the pair. Finally since we will be interested in using the AdS/CFT correspondence for $\mathcal{N}=4 \mathrm{SYM}$, our quarks and antiquarks will be substituted in the standard way by (massive) gauge bosons coming from Higgsing $U(N+1) \rightarrow U(N) \times U(1)$ through the inclusion of a probe D3 brane.

As a technical tool we will perform an analytical continuation to euclidean space. Thus we consider Wilson loops which form an angle $\theta$ in the longitudinal $t-y$ plane. The 'width' of the loops is along the transverse direction ' $x$ ' (see figure 1.1).

Explicitly we have to compute

$$
-2 i \int d^{2} x_{\perp} e^{i q x_{\perp}}\left\langle\frac{W_{1} W_{2}}{\left\langle W_{1}\right\rangle\left\langle W_{2}\right\rangle}-1\right\rangle
$$


as a function of the angle $\theta$, and after performing the calculation we analytically continue $\theta \longrightarrow-i \chi \sim-i \log \left(\frac{s}{2 m^{2}}\right)$ to obtain (1.1). In the next section we apply the AdS/CFT correspondence to calculate (1.2).

\section{SUPERGRAVITY CALCULATION}

According to the now standard prescription, a Wilson loop on the gauge theory side corresponds to a minimal surface string worldsheet in AdS bounded by this loop [8]. The correlation function of two Wilson loops, in the case when their separation is much larger than their transverse sizes, will be given by the exchange of supergravity fields between the two associated minimal string worldsheets. The calculation is technically completely analogous to the one of [9], the only change is a different $\theta$-dependent geometry of the loops, and the resulting interpretation as a gauge theory scattering amplitude. Due to the analytical continuation it turns out that the hiearchy of importance of various supergravity fields is different from that in [9].

The calculation involves two steps:

(1) determination of the couplings of the various supergravity fields to the string worldsheet,

(2) calculation of the interaction using the appropriate bulk to bulk Green's function.

The couplings are found by varying the Nambu-Goto action with respect to the background supergravity fields. The resulting contribution of the relevant field to the gauge theory scattering phase shift is then given by

$\frac{\left\langle W_{1} W_{2}\right\rangle}{\left\langle W_{1}\right\rangle\left\langle W_{2}\right\rangle}=\exp \left(\frac{1}{4 \pi^{2} \alpha^{\prime 2}} \int 4 d t_{1} d t_{2} \frac{d z d w}{z_{x} w_{x}} \frac{\delta S_{N G}}{\delta \psi}\left(t_{1}, z\right) G\left(x, x^{\prime}\right) \frac{\delta S_{N G}}{\delta \psi}\left(t_{2}, w\right)\right)$

where $\psi$ denotes a generic supergravity field. Using the symmetry properties of the AdS geometry one can show that the whole $\theta$ - (and consequently energy $s$-) dependence is encoded in the couplings. All the detailed properties of the Green's function only change the $a / L$ dependence of the amplitude.

We performed the calculations for the tachyonic scalars $s^{I}$, lightest modes of the dilaton, of the 2-form field and of the graviton. Similarly as in flat space the energy dependence turns out to be related to the spin of the exchanged particle.

The phase shift coming from the scalars behaves like $\delta_{K K} \sim(a / L)^{2}$. $(1 / \sin \theta) \sim(a / L)^{2} \cdot s^{-1}$. From the 2-form field $\delta_{B} \sim(a / L)^{4} \cdot(\cos \theta / \sin \theta) \sim$ $(a / L)^{4} \cdot s^{0}$ and for the graviton we get $\delta_{\text {grav }} \sim(a / L)^{6} \cdot\left(\cos ^{2} \theta / \sin \theta\right) \sim$ $(a / L)^{6} \cdot s^{1}$. Thus the graviton dominates at high energies. 
We would like to comment on the limitations of the above calculation. We considered only single particle exchange. This is legitimate in the large $N$ limit but for high enough energies may be inadequate. In fact analytically continuing to Minkowski signature within the AdS space one can check that the gravitational field produced by one of the worldsheets can be considered as a small perturbation around the background geometry only for $s^{2} \ll(L / a)^{7}$.

The surprising result from the gauge theory side is the $s^{1}$ dependence of the graviton contribution. In field theory we do expect such higher powers of $s$ to be supressed by unitarity corrections, but it is a difficult challenge to envisage how this would look like on the supergravity side, where there is no obvious process cancelling the attractive gravitonmediated interaction at high energies. Probably something very specific to string theory on AdS would have to intervene.

\section{Acknowledgments}

I would like to thank the organizers and participants of the Cargese ASI for a very stimulating school, and Robi Peschanski with whom these results were obtained. This work was supported in part by KBN grants 2P03B08614 and 2P03B01917.

\section{References}

[1] J. Maldacena, Adv. Theor. Math. Phys. 2 (1998) 231;

S.S. Gubser, I.R. Klebanov and A.M. Polyakov, Phys. Lett. B428 (1998) 105;

E. Witten, Adv. Theor. Math. Phys. 2 (1998) 253.

[2] O. Aharony, S.S. Gubser, J. Maldacena, H. Ooguri and Y. Oz, Large $N$ field theories, String Theory and Gravity, hep-th/9905111.

[3] R.A. Janik and R. Peschanski, High energy scattering and the AdS/CFT correspondence, hep-th/9907177.

[4] M. Rho, S.-J. Sin and I. Zahed, Elastic Parton-Parton Scattering from AdS/CFT, hep-th/9907126.

[5] O. Nachtmann, Ann. Phys. 209 (1991) 436.

[6] H. Verlinde and E. Verlinde, QCD at High Energies and TwoDimensional Field Theory, hep-th/9302104.

[7] G.P. Korchemsky, Phys. Lett. B325 (1994) 459.

[8] J. Maldacena, Phys. Rev. Lett. 80 (1998) 4859;

S.-J. Rey and J. Yee, Macroscopic strings as heavy quarks in large $N$ gauge theory and anti-de Sitter supergravity, hep-th/9803001.

[9] D. Berenstein, R. Corrado, W. Fischler and J. Maldacena, Phys. Rev. D59 (1999) 105023. 